\title{
A FLEXIBLE PROTOCOL COMMUNICATIONS APPROACH FOR A SUPERVISORY SYSTEM
}

\author{
R. Valle-Alarcón, J. Serrat-Fernández \\ Dept. Teoría de la Señal y Comunicaciones \\ E.T.S.E. de Telecomunicación, Univ. Politec. Catalunya \\ P.O. Box 30002 - 08080 Barcelona (Spain) \\ Phone: 34-3-2046551
}

\section{Abstract}

This paper deals with the communication protocol intended to control up to 1016 devices of a new supervisory system. It has been conceived in two complementary phases. The first one (Cycle-A) is intended to inform the Central Station of any event detected by the installed devices in less than two seconds. The second one allows to set up a link between the Central Station and a selected Local Station in order to request more information or to act on its devices for command or programming purposes. The dialog between the Central Station and the Local station takes place on a two wire communication channel, also used to supply de current from the Central to all Local Stations.

\section{Introduction}

Present days supervisory and control systems make use of microprocessor or microcomputer technologies in order to ful-fill general or more site dependent requirements. Both kind of them must be taken into account in order to conceive the final-product. This has been the approach used by the authors to conceive the system architecture and the communication protocol outlined in this paper. Let us to make a few comments on two of such requirements, say the system cost and flexibility, in order to show the importance of this point of view.

The system cost is strongly dependent on the price per unit length of the cable used in the instalation. This is because the system is constituted by several devices spread out in areas of several thousands of square meters. The way to reduce this cost is to use a two wi re cable of little diameter without screen. In order to use a two wire cable, it will be nece ssary to carry both the information signals and the dc power supply on the same physical medium. Regardless mechanical strength, the wi re diameter will be lower bounded by the product of the maximum number of devices present and the maximum power consumed by each one. Mo reover, if it is necessary to avoid the screen special attention will be paid to protect the communication system against electromagnetic interference.

The system flexibility must be understood as its ability to be accomodated to different user needs and to allow the evolution to support new devices and/or applications. Therefore, it must be possible to instal the system with a number of devices (detection and command points) ranging from a few tens to say a thousand, without cost penalties. The detection points can be used in fire or intrusion environments. The detection devices can be simply of two states (on-off) or more complex like the analog ones.

On the other hand, the system will be fle xible and low cost if its hardware and software components are designed in a modular way. Then it will be possible to tailor it to the user needs or to modify, add, or replace modules without afecting the other subsystems.

The following paragraphs are devoted to describe a system conceived under the above mentioned criteria. First of all we give an overview on its architecture to esplain then the communication protocol, a key item to grant it the required properties.

\section{The system architecture}

The system is constituted by a Central sta tion (CS) and several Local stations (LS) spread out in the area to be covered by the se curity service. The network topology can be either a tree or a ring. In both cases the LS are conected directly to the CS, therefore the communication channel is a bidirectional bus. Figure 1 show both structures

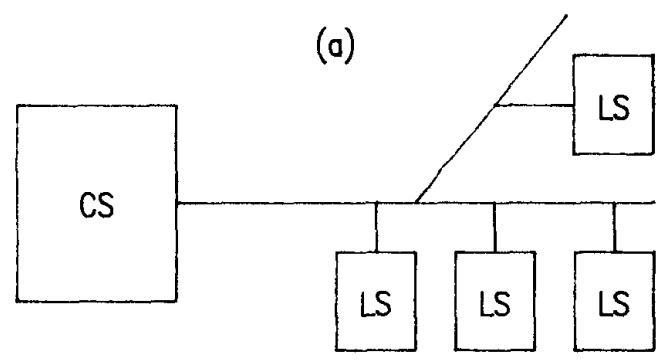

(b)

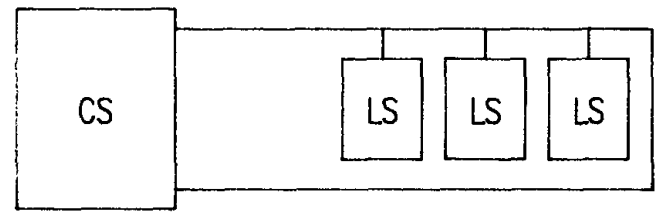

Figure 1.- Different network topologies. (a) tree: (b) ring. CS: Central Station (LS: Local Station.

The communication channel is a two wire transmission line intended to carry orders and answers to/from the LS and the dc power supply. The system can be eqquiped with up to $127 \mathrm{LS}$. Each LS can be intended to control up to 8 detection points or 8 command points. Detection and command functions can be supported in the same LS. Therefore, combining the existing po ssibilities concerning the type of function and the type of devices installed in each LS, it is possible to configure up to 8 different types of LS. Notice that the maximum number of devices is 1016 . 


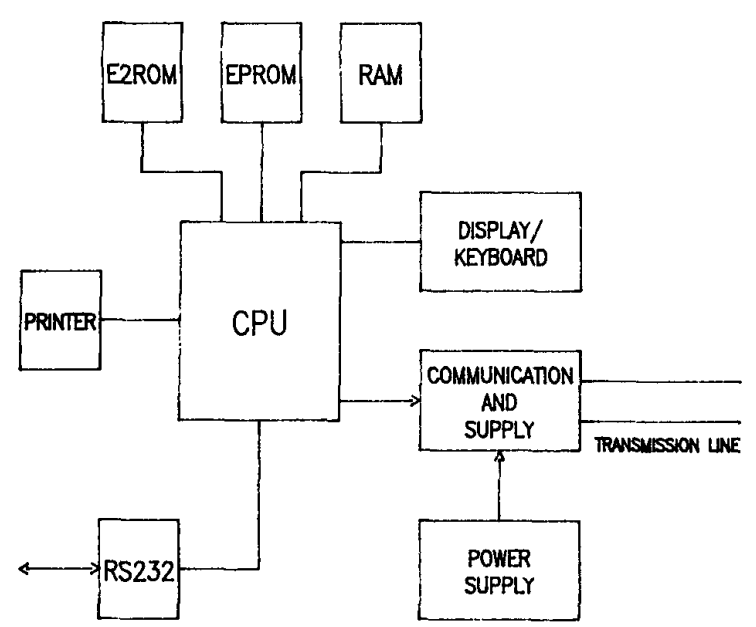

Figure 2.- Functional block diagram of the Cen tral station.

The CS is built around a Central Processing Unit (CPU), accessed by several peripheral devices. Figure 2 shows the block diagram of the CS. EPROM E2ROM and RAM memories are used to store the main program and its subroutines as well as the data comming from the Ls or from the user interface. The user is allowed to introduce or to get data from the CS for programming purposes or simply to know about the system state. This is done directly through the display/keyboard unit or by a personal com puter through the RS232 interface. The commun $\bar{i}$ cation and supply unit is intended to deal with the electrical signals in the physical co nexion medium. Finally, a printer unit allows a permanent register of the detected events or of the remote device states.

The LS are built around a microcomputer (the control unit) which stores the software of all its characteristic functions. The main differences between different types of LS arise from the input/output interfaces. Figure 3 (a) shows a LS with 8 detection points whilst figure $3(\mathrm{~b})$ is the same but for 8 command points. The data from the detection points are introduced into the control unit by means of a multiplexer, whilst the orders are sent through appropriate drivers to relays. Each LS has assigned a unique identification code set by switches on the same card board. This code is used for addressing purposes in the communication process. As with the CS, the communication and supply unit is intended to insert or extract the corresponding electrical signals to/from the two wire line.

\section{The communication protocol}

The communication protocol between the CS and the LS has been structured in two levels, namely the Physical level and the link level. Both are described as they have been implemented in a demostration prototype.
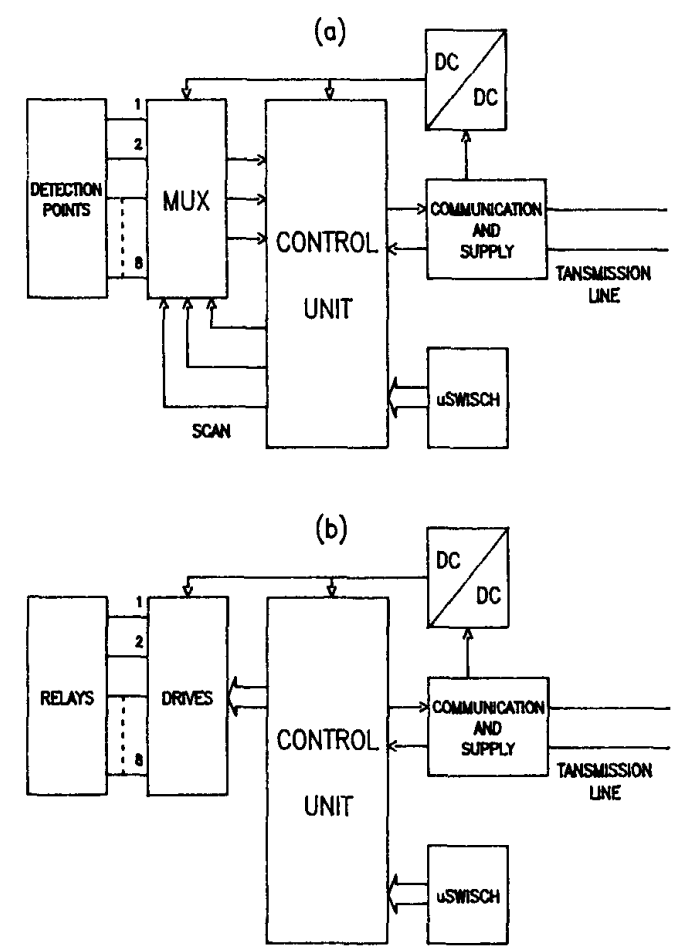

Figure 3.- Functional block diagram of a Local Station eqquiped with detection points (a) and command points (b).

Physical leve1

The physical level deals with aspects related to the electrical signals intended to ca rry the information flux between the involved parties.

The information is supplied and transmitt ed asynchronously over the half dupplex channel at 1200 Band rate. The digital train is then modulated (ON-OFF Keying) using a $150 \mathrm{KHz} \mathrm{ca}-$ rrier. The modulated carrier is inserted at 15 volts, by means of the feeding device, into the transmission line with the output of a 24 volts power supply.

\section{Link level}

The link level deals with the information structure and with the synchronization and pro cedures between the $C S$ and all the involved $L \bar{S}$. Moreover, it provides an error free communication channel.

The access to the communication channel is granted to each LS always under the control of the CS. This is carried out in two different ways. Therefore we have identified two co mmunication processes, namely Cycle-A and $\mathrm{CY}^{-}$ cle-B and several tasks into them.

The Cycle-A is intended to get a periodic reporting from all local stations (task SAl) and to know, when required, the identification code and type of all presently installed LS

(task SA2). In this case the access is done in 
a TDMA basis: The CS transmits a unique bit pattern with a twofold application; it is the begining of a 1,28 seconds TDMA frame and it specifies what task must be done (SA1 or SA2). Each LS is allowed to answer into a $10 \mathrm{~ms}$ time slot with a delay from the reference which depends on the code word assigned to it. This ti me slot is wide enough to prevent collisions between adjacent LS. The SAI frames are repeat ed every 2 seconds as represented in figure 4 .

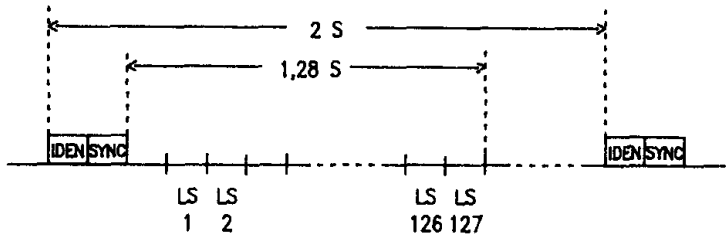

Figure 4.- Communication protocol - Cycle A.

Therefore, the CS can know the presence of any event in the system with a maximum delay of 2 seconds.

The Cycle-B is intended for the following purporses:

- Selective request (task SB1)

- Order to a command device (task SB2)

- Order to a detection device (task SB3)

- Programming of detection devices (task SB4)

- Programming of command devices (task SB5)

This five tasks are carried out by selective polling between the SAl frames. Moreover, the information transfer is protected against transmission errors by means of a twofold auto matic repeat request procedure. Figure 5 shows the code words transmitted in both senses during each task. Every code word is 9 bits long. The first bit is used to distinguish between an address (DIR) and the other fields. The last bit is the pasity bit. Therefore there are 7 bits to code addresses, inquires, answers or information.

\begin{tabular}{|c|c|c|}
\hline TASK & FROM-TO & CODEWORDS \\
\hline \multirow{3}{*}{ SB 1} & $\mathrm{CS} \rightarrow \mathrm{LS}$ & DIR - OPT 1 \\
\hline & $\mathrm{LS} \rightarrow \mathrm{CS}$ & DIR - OPRI - INF \\
\hline & $\mathrm{CS} \rightarrow \mathrm{LS}$ & DIR - VAL \\
\hline \multirow{4}{*}{ SB2 } & $\mathrm{CS} \rightarrow \mathrm{LS}$ & DIR - OPT2 - INF \\
\hline & $\mathrm{LS} \rightarrow \mathrm{CS}$ & DIR - OPR2 - INF \\
\hline & $\mathrm{CS} \rightarrow \mathrm{LS}$ & DIR - VAL \\
\hline & $\mathrm{LS} \rightarrow \mathrm{CS}$ & DIR - VAL \\
\hline \multirow{4}{*}{ SB3 } & $(\mathrm{CS} \rightarrow \mathrm{LS}$ & DIR - OPT 3 \\
\hline & $\mathrm{LS} \rightarrow \mathrm{CS}$ & DIR - OPR 3 \\
\hline & $\mathrm{CS} \rightarrow \mathrm{LS}$ & DIR - VAL \\
\hline & $\mathrm{LS} \rightarrow \mathrm{CS}$ & $D I R-V A L$ \\
\hline \multirow{4}{*}{ SB 4} & $\mathrm{CS} \rightarrow \mathrm{LS}$ & DIR - OPT $4-$ INF \\
\hline & $\mathrm{LS} \rightarrow \mathrm{CS}$ & $D I R-O P R 4-I N F$ \\
\hline & $\mathrm{CS} \rightarrow \mathrm{LS}$ & DIR - VAL \\
\hline & $\mathrm{LS} \rightarrow \mathrm{CS}$ & DIR - VAL \\
\hline
\end{tabular}

TASK

FROM-TO

CODEWORDS

$\left\{\begin{array}{l}\mathrm{CS} \rightarrow \mathrm{LS} \\ \mathrm{LS} \rightarrow \mathrm{CS} \\ \mathrm{CS} \rightarrow \mathrm{LS} \\ \mathrm{LS} \rightarrow \mathrm{CS}\end{array}\right.$

DIR - OPT5 - INF

DIR - OPR5 - INF

DIR - VAL

DIR - VAL

Figure 5.- Communication protocol - Cycle B.

DIR : address of the LS

OPTi: inquire or command during task SBi

OPRi: answer or echo during task SBi

VAL : validation of the current task

$I N F$ : information

\section{Conclusions}

A new supervisory system has been conceived in order to keep a high degre of software and hardware flexibility. It can be used in $f_{\underline{i}}$ re or intrusion security services being able to control up to 1016 different devices in a few seconds. Moreover, these devices can be in dividually programmed and linked from the central Station to satisfy the most diverse user needs by means of an user friendly interface or by a high level program from a PC. 